\title{
Sur la régularité des ondes progressives à la surface de l'eau
}

\section{Walter Craig Ana-Maria Matei}

\section{Résumé}

Il a été établi par H. Lewy (1952) qu'une surface libre hydrodynamique qui est au moins $C^{1}$ dans un voisinage d'un point $q$ situé à la surface libre, est automatiquement $C^{\omega}$, éventuellement dans un voisinage plus petit de $q$. Ce résultat local est un exemple qui précedait la théorie dévélopée par D. Kinderlehrer, L. Nirenberg et J. Spruck (1977 - 79) demontrant que dans beaucoup de cas, des surfaces libres ne peuvent pas être d'une régularité arbitraire, et en particulier ils existent $m, \alpha$ tels que, si la surface en question est $C^{m, \alpha}$, alors automatiquement elle est $C^{\omega}$. Nous allons présenter leurs methodes de transformation de Legendre/hodographe partielle, et les prolongements de ces methodes aux problemes en plusieures dimensions et avec la tension superficielle.

\section{Les équations des surfaces libres}

Les équations classiques du mouvement d'un fluide ideal à surface libre dans une configuration stationnaire, sont posées dans un domaine $R:=\left\{x^{\prime} \in \mathbb{R}^{n-1},-h<\right.$ $\left.x_{n}<\eta\left(x^{\prime}\right)\right\}$, et la surface libre est $\Sigma:=\left\{x_{n}=\eta\left(x^{\prime}\right)\right\}$. Posée dans les coördonnées Euleriennes, la vitesse du fluide est donné par un champs de vecteurs $u(x)$ defini sur $R$; etant un fluide incompressible et irrotationel, il satisfait

$$
\nabla \cdot u=0, \quad \nabla \wedge u=0
$$

dans le domaine $R$. Ce qui entraine que $u(x)=\nabla \Phi(x)$ pour une fonction de potentiel $\Phi(x)$ telle que dans le domaine $R$

$$
\Delta \Phi(x)=0 .
$$

Les conditions aux bords pour $\Phi(x)$ sont

$$
N \cdot \nabla \Phi=0 \quad \text { sur la frontière } \partial R ;
$$

Cette recherche a été soutenue par le NSF, le NSERC, le Programme de Chairs de Recherche du Canada, et le CNRS sous le Programme des Postes Rouges 2003 
et en plus sur la surface libre,

$$
\frac{1}{2}|\nabla \Phi|^{2}+g \eta=\frac{c^{2}}{2}
$$

où la constante de Bernoulli $c^{2} / 2$ est en terme de la vitesse $c$ de la solution. Il se peut que les effets de la tension superficielle soient inclus dans le problème, ce qui change la condition au bord (3) en

$$
\frac{1}{2}|\nabla \Phi|^{2}+g \eta+\sigma H(\Sigma)=\frac{c^{2}}{2},
$$

où $H(\Sigma)$ est la courbure moyenne de la surface libre $\Sigma$,

$$
H(\Sigma)=-\nabla \cdot\left(\frac{\nabla \eta}{\sqrt{1+(\nabla \eta)^{2}}}\right)
$$

L'objet de ce travail est d'étudier la régularité de la surface libre $\Sigma$. Ce genre de problème est illustré par un résultat classique de Hans Lewy.

Théorème 1. [5] (H. Lewy, Proceedings AMS, 1952) Soit $n=2$, et supposons que $\eta\left(x_{1}\right)$ et $\Phi\left(x_{1}, x_{2}\right)$ satisfont les équations (1),(2),(3), et qu'elles sont de classe $C^{1}\left(B_{r}(q) \cap(R \cup \Sigma)\right)$ au voisinage d'un point $q \in \Sigma$. Alors a priori

$$
(\eta, \Phi) \in C^{\omega}\left(B_{\frac{r}{2}}(q)\right)
$$

Ce résultat est optimal, dans le sens qu'il existe des solutions du problème (1),(2),(3) qui sont Lipschitzienne mais ne sont pas $C^{1}$, notamment les ondes de Stokes de forme extremale. Ces ondes sont les points extremes des branches de bifurcation des ondes progressives, et elles possèdent des crètes maximalles en forme d'une singularité Lipschitzienne avec angle d'ouverture $2 \pi / 3$.

Nous précisons la question : est-ce que les ondes progressives possèdent une propriété analogue de régularité a priori, ou bien pour les conditions (3) en plusieures dimensions $n \geq 3$, ou bien dans le cas avec l'inclusion de la tension superficielle (4), pour $n=2$ et pour $n \geq 3$ ?

\section{Quelques résultats récents}

Pour répondre à cette question, la première etape est un résultat de prolongement du théorème de Lewy pour inclure la tension superficielle.

Théorème 2. [6] (A.-M. Matei, CRAS, 2002) Soit $n=2$, et supposons que $\sigma>0$ dans le problème (1) imposant les conditions (2)(4) à la surface libre. Alors toute solution $(\eta, \Phi)$ qui est au moins $C^{2}\left(B_{r}(q) \cap(R \cup \Sigma)\right)$, à un point $q \in \Sigma$ est a priori $C^{\omega}$.

La démonstration utilise l'existence d'une fonction $\Psi(x)$ qui est la conjugée harmonique de $\Phi(x)$, ce qui dépend fortement du fait que le problème est en deux dimensions. Cependant la démonstration est liée à la technique dévélopée dans les articles [2] de D. Kinderlehrer et L. Nirenberg, et [3][4] de D. Kinderlehrer, L. Nirenberg et J. Spruck, qui est effectivement independente de la dimension. Un prolongement de ce théorème est le suivant; 
Théorème 3. (W. Craig et A.-M. Matei, 2002) Dans le cas ou $n \geq 3$ supposons que $\sigma>0$. Alors toute solution de l'équation (1) qui satisfait les conditions à la surface libre (2)(4) qui est localement $C^{2, \alpha}$, est a priori $C^{\omega}$.

Ce qu'on voudrait savoir est si, dans le cas du problème $n \geq 3$ et $\sigma=0$, une propriété analogue est valable, ce qui réprésente un travail en cours. Dans une autre direction, il est possible que des mathématiciens puissent en profiter pour étudier les formes possibles des singularités des crêtes des ondes progressives en trois dimensions, ce qui est une question completement ouverte, autant que nous sachions.

Prenons un problème qui généralise ces deux problèmes de surfaces libres à conditions de Neumanns.

$$
\begin{aligned}
\Delta \Phi(x) & =0, \quad x \in R ; \\
\partial_{N} \Phi(x) & =0 \quad x \in \Sigma \subseteq \partial R .
\end{aligned}
$$

Sur la surface libre $\Sigma$ on impose aussi la deuxième condition

$$
G(x, \nabla \Phi)=0
$$

Pour le problème des ondes progressives à la surface de l'eau on a

$$
G=\frac{1}{2}|\nabla \Phi|^{2}+g x_{n}-\frac{c^{2}}{2}
$$

Quand on modifie le problème avec la tension superficielle, la forme générale des connditions aux bords devient

$$
G(x, \nabla \Phi)+\sigma H(\Sigma)=0 .
$$

Par comparaison, une classe parallèle de problèmes où les conditions Dirichlets aux bords jouent un rôle central, est

$$
\begin{aligned}
\Delta \Psi(x) & =0, \quad x \in R ; \\
\Psi(x) & =0 \quad x \in \Sigma \subseteq \partial R .
\end{aligned}
$$

La deuxième condition qu'on impose sur la surface libre $\Sigma$ est

$$
F(x, \nabla \Psi)=0 .
$$

Nous allons considérer seulement les nonlinéarités $F$ et $G$ qui sont $C^{\omega}$ dans les deux variables .

En dimension $n=2$ les problèmes (9) et (6) sont présque équivalent, au moins sur la question de la régularité des solutions. En particulier, la fonction $(\Phi+i \Psi)\left(x_{1}+\right.$ $\left.i x_{2}\right)$ est analytique, et quand $\partial_{N} \Phi=0$ sur une courbe $\Sigma \subseteq \partial R$, alors $\partial_{T} \Psi=$ 0 sur $\Sigma$. Modulo une constante, on peut supposer que $\Psi=0$ sur $\Sigma$. En plus, $\nabla \Phi=J \nabla \Psi$, alors mettant $F(x, \nabla \Psi)=G(x, J \nabla \Psi)$ on établit une transformation du problème (6) en (9), au moins dans la situation dans laquelle les conjugées harmoniques partage la même régularité jusqu'au bord $\Sigma$, ce qui est le cas dans les classes $C^{k, \alpha}$ quand $k+\alpha \geq 1$. 
En dimensions $n \geq 3$ il y a une théorie générale pour des problémes du type (9) (10). Mais il exite un contre-exemple [2] des énoncés en tel généralité pour des problèmes du type (6)(7) en dimensions $n \geq 3$. Mettons

$$
G(x, \nabla \Phi)=|\nabla \Phi|^{2}-1
$$

et considérons la fonction harmonique $\Phi(x)=x_{1}$. La frontiére libre sera $\Sigma=\left\{x_{n}=\right.$ $\left.\eta\left(x_{2}, \ldots, x_{n-1}\right)\right\}$. Il est clair que $|\nabla \Phi|^{2}=1$ et que $\nabla \Phi=(1,0) \perp N=\left(0,-\partial_{x^{\prime}} \eta, 1\right)$, alors les conditions aux bords sont satisfaites. Pourtant la fonction $\eta$ peut être d'une régularité arbitraire, et donc un énoncé général comme celui du Théorème 1 n'est pas possible.

\section{La démonstration du Théorème 3}

Rappelons le problème (6)(8), pour une fonction de potentiel $\Phi$ qui satisfait des conditions aux bords nonlinéaires sur une portion de la frontière $\Sigma \subseteq \partial R$. dont en particulier la tension superficielle est inclue. Supposons qu'il y a un point $q \in \Sigma$ tel que localement au voisinage de $q$ le potential et la surface libre satisfont $\Phi, \Sigma \in C^{2, \alpha}$.

On construit ensuite une fonction auxiliaire $\rho(x)$ qui servira comme une conjuguée harmonique, même si en dimension plus élévée que deux elle ne possède pas toutes les propriétés d'une vraie conjuguée;

$$
\begin{aligned}
\Delta \rho(x) & =0, \quad \text { dans } \quad R \cap B_{r}(q) \\
\rho(x) & =0, \quad \text { sur } \quad \Sigma \cap B_{r}(q) \\
\rho(x) & =g(x) \leq 0, \quad \text { sur } \quad \partial\left(R \cap B_{r}(q)\right) \backslash \Sigma .
\end{aligned}
$$

On suppose que $g \in C^{\infty}$ et simplement qu'elle n'est pas identiquement nulle. Le principe de maximum implique $\rho(x)<0$ dans $R \cap B_{r}(q)$, et le lemme de Hopf implique que $N \cdot \nabla \rho(x)>0$ sur $\Sigma$. En plus, $\rho \in C^{2, \alpha}\left(\overline{R \cap B_{r}(q)}\right)$. Nous performons un changement de variables dans les équations (6)(8) (12), en utilisant la fonction

$\rho(x)$, pour transformer le domaine $R$ localement en un demi-espace, et la surface libre $\Sigma$ en un sous-ensemble d'un hyperplane. Plus précisement, supposons que $q=0$, que l'espace tangent $T_{q}(\Sigma)$ du $\Sigma$ au point $q$ est $\left\{\left(x^{\prime}, 0\right): x^{\prime} \in \mathbb{R}^{n-1}\right\}$, et mettons $y_{n}=\rho(x)$. C'est-à-dire

$$
x=\left(x_{1}, \ldots, x_{n}\right)=\left(x^{\prime}, x_{n}\right) \mapsto y=\left(x^{\prime}, \rho(x)\right)=\left(y^{\prime}, y_{n}\right) .
$$

Cette transformation est bien définie localement, car la condition de non dégénérescence au point $q$ est

$$
\partial_{x_{n}} \rho(q)=N \cdot \nabla \rho(q)>0 .
$$

Nous appelons la transformation inverse $x_{n}=\tau(y)$. Elle est l'analogue de la transformation du 'type $p=0$ ' dans les travaux de D. Kinderlehrer, L. Nirenberg et J. Spruck [2, 3, 4].

Effectuons la transformation sur l'EDP en question. On a

$$
\tau\left(x^{\prime}, \rho(x)\right)=x_{n},
$$


alors

$$
\partial_{y_{\alpha}} \tau+\partial_{y_{n}} \tau \partial_{x_{\alpha}} \rho=0, \quad \partial_{y_{n}} \tau \partial_{x_{n}} \rho=1
$$

ceci implique que

$$
\partial_{x_{\alpha} \rho}=-\frac{\partial_{y_{\alpha}} \tau}{\partial_{y_{n}} \tau}, \quad \partial_{x_{n}} \rho=\frac{1}{\partial_{y_{n}} \tau} .
$$

Mettons $\Phi(x)=\Xi(y)$ qui sert pour définition de la nouvelle fonction $\Xi(y)$. On exprime ces dérivées du premier ordre en fonction des variables $y$

$$
\partial_{x_{\alpha}} \Phi(x)=\partial_{y_{\alpha}} \Xi(y)+\rho_{x_{\alpha}} \partial_{y_{n}} \Xi(y), \quad \partial_{x_{n}} \Phi(x)=\rho_{x_{n}} \partial_{y_{n}} \Xi(y) \text {; }
$$

ce qui donne aussitôt une expression en variables $y$ vu les relations (16) pour les dérivées de $\rho(x)$ en terme de $\tau(y)$. Les expressions pour les dérivées d'ordre deux suivent dans la même manière;

$$
\begin{aligned}
\partial_{x_{\alpha}}^{2} \Phi & =\partial_{y_{\alpha}}^{2} \Xi+\rho_{x_{\alpha}}^{2} \partial_{y_{n}}^{2} \Xi+2 \rho_{x_{\alpha}} \partial_{y_{\alpha}} \partial_{y_{n}} \Xi+\rho_{x_{\alpha} x_{\alpha}} \partial_{y_{n}} \Xi, \\
\partial_{x_{n}}^{2} \Phi & =\rho_{x_{n}}^{2} \partial_{y_{n}}^{2} \Xi+\rho_{x_{n} x_{n}} \partial_{y_{n}} \Xi .
\end{aligned}
$$

L'équation de Laplace pour la fonction $\Phi$ est transformée en une équation elliptique pour $\Xi(y)$ à coefficients en terme de $\tau(y)$;

$$
\begin{aligned}
0=\Delta_{x} \Phi & =\Delta_{y^{\prime}} \Xi+\left|\nabla_{x} \rho\right|^{2} \partial_{y_{n}}^{2} \Xi+2 \sum_{\alpha<n} \partial_{x_{\alpha}} \rho \partial_{y_{\alpha}} \partial_{y_{n}} \Xi \\
& =\Delta_{y^{\prime}} \Xi+\frac{1+\left|\partial_{y^{\prime}} \tau\right|^{2}}{\partial_{y_{n}} \tau^{2}} \partial_{y_{n}}^{2} \Xi-2 \sum_{\alpha<n} \frac{\partial_{y_{\alpha}} \tau}{\partial_{y_{n}} \tau} \partial_{y_{\alpha}} \partial_{y_{n}} \Xi
\end{aligned}
$$

Deuxièmement, calculons l'effet de la transformation définie au moyen de la fonction $\rho(x)$, sur l'équation (12) pour $\rho$ même [2];

$$
0=\Delta_{x} \rho=\left(\sum_{\alpha<n}-\left(\frac{\tau_{y_{\alpha}}}{\tau_{y_{n}}}\right)_{y_{\alpha}}+\frac{\tau_{y_{\alpha}}}{\tau_{y_{n}}}\left(\frac{\tau_{y_{\alpha}}}{\tau_{y_{n}}}\right)_{y_{n}}\right)+\frac{1}{\tau_{y_{n}}}\left(\frac{1}{\tau_{y_{n}}}\right)_{y_{n}} .
$$

Proposition 4. (D. Kinderlehrer et L. Nirenberg [2]) L'équation (19) pour la fonction $\tau(y)$ est elliptique nonlinéaire.

Les conditions aux bords sur $\Sigma$ sont aussi transformées dans les nouvelles variables. Tout d'abord, $\Sigma=\left\{x_{n}=\tau\left(y^{\prime}, 0\right)\right\}$, alors

$$
\begin{aligned}
N_{y} & =\frac{1}{\sqrt{1+\left|\nabla_{y^{\prime}} \tau\right|^{2}}}\left(-\nabla_{y^{\prime}} \tau, 1\right), \\
H(\Sigma) & =\frac{1}{n-1} \sum_{\alpha, \beta<n} \frac{\left(\left(1+\left|\nabla_{y^{\prime}} \tau\right|^{2}\right) \delta_{\alpha \beta}-\tau_{y_{\alpha}} \tau_{y_{\beta}}\right)}{\left(1+\left|\nabla_{y^{\prime}} \tau\right|^{2}\right)^{3 / 2}} \tau_{y_{\alpha} y_{\beta}} .
\end{aligned}
$$

Les conditions

$$
\begin{aligned}
\partial_{N} \Phi & =0 \\
G(x, \nabla \Phi) & +\sigma H(\Sigma)=0 \text { sur } \Sigma
\end{aligned}
$$


se transforment en

$$
\begin{aligned}
0 & =\partial_{N_{x}} \Phi=\frac{1}{\sqrt{1+\left|\nabla_{y^{\prime}} \tau\right|^{2}}}\left(-\sum_{\alpha<n} \tau_{y_{\alpha}}\left(\Xi_{\alpha}-\frac{\tau_{y_{\alpha}}}{\tau_{y_{n}}} \Xi_{n}\right)+\frac{1}{\tau_{y_{n}}} \Xi_{n}\right) \\
0 & =G+\sigma H(\Sigma)=K\left(y^{\prime}, \tau, \nabla_{y} \tau, \nabla_{y} \Xi\right)+\sigma H(\Sigma)
\end{aligned}
$$

qui sont imposées sur un ouvert du sous-espace linéaire $S \subseteq\left\{\left(y^{\prime}, 0\right) \in \mathbb{R}^{n}\right\}$, où $K\left(y^{\prime}, \tau, \nabla_{y} \tau, \nabla_{y} \Xi\right)=G\left(\left(y^{\prime}, \tau\right),\left(\nabla_{y^{\prime}} \Xi-\left(\Xi_{n} / \tau_{y_{n}}\right) \nabla_{y^{\prime}} \tau,\left(1 / \tau_{y_{n}}\right) \Xi_{n}\right)\right)$.

Théorème 5. Le système nonlinéaire après la transformation (19) (20) (23) est elliptique et coercive.

Corollaire 6. Quand la solution $(\Phi, \rho)$ atteint un niveau minimal de régularité, essentiellement quand $(\Phi, \Sigma) \in C^{2, \alpha}$, alors la solution est automatiquement $C^{\omega}$.

Démonstration : Nous avons pris des coordonnées telles que $q \mapsto 0$ et telles que l'image de $\Sigma$ est un ouvert dans le sous-espace $\left\{\left(y^{\prime}, 0\right): y^{\prime} \in \mathbb{R}^{n-1}\right\}$. Nous pouvons aussi supposer que l'espace tangent $T_{q}(\Sigma) \subseteq\left\{\left(x^{\prime}, 0\right): x^{\prime} \in \mathbb{R}^{n-1}\right.$, et que $\partial_{x_{n}} \rho(0)=$ $\partial_{N} \rho(0)=1$. Alors $\tau_{y_{n}}(0)=1$ et $\nabla_{y^{\prime}} \tau(0)=0, \Xi_{y_{n}}(0)=\Phi_{x_{n}}(q)=0$. Le système linéarisé posé pour les variations $\xi=\delta \Xi$ et $T=\delta \tau$ prend le forme

$$
\Delta_{y} \xi=0, \quad \Delta_{y} T=0
$$

et la partie principale des conditions aux bords est

$$
-\nabla_{y^{\prime}} \Xi(0) \cdot \nabla_{y^{\prime}} T+\partial_{y_{n}} \xi=0, \quad \sigma \Delta_{y^{\prime}} T=0 \quad \text { sur } \quad S .
$$

Il est clair que (24) est elliptique. Pourvu que la constante $\sigma \neq 0$ il n'existe pas de solutions nontriviales du système $(24)(25)$ qui tend vers zéro quand $y_{n} \rightarrow-\infty$; ce qui effectivement implique la propriété de coercivité.

Quand le coefficient de la tension superficielle s'annule, $\sigma=0$, l'opérateur linéarisé s'exprime

$$
\left(\begin{array}{cc}
\partial_{y_{n}} & -\Xi_{y^{\prime}} \cdot \partial_{y^{\prime}} \\
\partial_{p^{\prime}} G(\cdot) \cdot \partial_{y^{\prime}}+\partial_{p_{n}} G(\cdot) \partial_{y_{n}} & \partial_{x_{n}} G(\cdot)
\end{array}\right)\left(\begin{array}{l}
\xi \\
T
\end{array}\right)=0
$$

où $G(\cdot)=G\left(0, \Xi_{y^{\prime}}, 0\right)$. Dans le problème hydrodynamique, nous avons

$$
\partial_{p^{\prime}} G\left(0, \Xi_{y^{\prime}}, 0\right)=\Xi_{y^{\prime}}, \quad \partial_{p_{n}} G\left(0, \Xi_{y^{\prime}}, 0\right)=\Xi_{y_{n}}=0,
$$

qui ne donne pas lieu désormais à un problème du type coercif dans le sense classique.

Une alternative est de se servir d'une autre transformation du problème, qui est la transformation de Legendre partielle. Pour bien la décrire, considerons le problème alterné suivant, qui est équivalent au problème (6)(7) :

$$
\begin{aligned}
\Delta \Phi & =0, & & \Delta \rho=0, \quad x \in R ; \\
\rho & =0, & & \nabla \Phi \cdot \nabla \rho=0, \quad x \in \Sigma \\
G(x, \nabla \Phi) & =0 . & &
\end{aligned}
$$


Au lieu de discuter la régularité des solutions de (27), on voudrait présenter un problème différent, pour lequel on peut appliquer la théorie de D. Kinderlehrer, L. Nirenberg et J. Spruck;

$$
\begin{aligned}
\Delta \Phi=0, \quad x \in R \\
\partial_{x_{n}} \Phi=0, \quad G(x, \nabla \Phi)=0, \quad x \in \Sigma,
\end{aligned}
$$

où $\partial_{x_{n}}$ est un champ de vecteurs fixe.

Théorème 7. Soit $\Phi, \Sigma$ une solution de (28) qui satisfait $\Phi, \Sigma \in C^{2, \alpha}$. Supposons pour $q \in \Sigma$ que

$$
\partial_{x_{n}}^{2} \Phi(q) \neq 0, \quad \partial_{x_{n}} G(q, \nabla \Phi(q)) \neq 0 .
$$

Alors a priori, $\Phi, \Sigma$ sont de classe $C^{\omega}$ localement au voisinage de $q$.

Nous remarquons que le cas hydrodynamique entraine un choix de $G=\frac{1}{2}|\nabla \Phi|^{2}+$ $g \cdot x-\frac{c^{2}}{2}$, où le vecteur $-g=-\left(g_{1}, \ldots, g_{n}\right)$ indique la direction de gravité. L'hypothèse est valable justament quand $\partial_{x_{n}}(g \cdot x)=g_{n} \neq 0$.

Démonstration : Suivant les références [2][3][4], on applique une transformation de Legendre partielle, qui s'écrit

$$
\begin{aligned}
x & =\left(x^{\prime}, x_{n}\right) \mapsto y=\left(x^{\prime},-\partial_{x_{n}} \Phi\right) \\
\Psi(y) & =\Psi(x)-x_{n} \partial_{x_{n}} \Phi(x)
\end{aligned}
$$

Cette transformation est bien définie dans un voisinage de $R$ qui contient $q \in \Sigma$ sa frontière, à cause de la condition de nondégénérescence $\partial_{x_{n}}^{2} \Phi(q) \neq 0$. . Le résultat de la transformation est

$$
\begin{aligned}
x_{n} & =\partial_{y_{n}} \Psi \\
\partial_{x_{\alpha}} & =\partial_{y_{\alpha}}-\frac{\Psi_{y_{\alpha} y_{n}}}{\Psi_{y_{n} y_{n}}} \partial_{y_{n}}, \quad \partial_{x_{n}}=\frac{1}{\Psi_{y_{n} y_{n}}} \partial_{y_{n}},
\end{aligned}
$$

Il est un fait établi [2] qu'une équation elliptique en variables $x$ est transformée en une équation elliptique en variables $y$. En particulier,

$$
\begin{aligned}
0 & =\Delta_{x} \Phi(x) \\
& =-\frac{1}{\Psi_{y_{n} y_{n}}}+\sum_{\alpha<n}\left(\Psi_{y_{\alpha} y_{\alpha}}-\frac{\Psi_{y_{\alpha} y_{n}}^{2}}{\Psi_{y_{n} y_{n}}}\right) .
\end{aligned}
$$

Commme prévu, la condition $\partial_{x_{n}} \Phi=0$ implique que $\Sigma$ se transforme dans un sousensemble d'une variété fixe; $S \subseteq\left\{y=\left(y^{\prime}, 0\right)\right\} \subseteq \mathbb{R}^{n}$. La deuxième condition aux bords dans les nouvelles variables devient

$$
G\left(\left(y^{\prime}, \partial_{y_{n}} \Psi\right), \partial_{y^{\prime}} \Psi, y_{n}\right)=0, \quad \text { sur } \quad S \subseteq\left\{\left(y^{\prime}, 0\right)\right\}
$$

Nous remarqons que par contre la propriété de coercivité n'est pas conservée par des transformations de ce genre. Pour le problème (28), la linéarisation des conditions aux bords (33) est

$$
\partial_{x_{n}} G(\cdot) \partial_{y_{n}} \psi+\partial_{p^{\prime}} G(\cdot) \partial_{y^{\prime}} \psi=0
$$


où $\delta \Psi=\psi$ et $G(\cdot)=G\left(0, \partial_{y^{\prime}} \Psi(0), 0\right)$. Ceci donne une condition coercive quand $\left(\partial_{x_{n}} G(\cdot)\right) \neq 0$, ce qui es l'énoncé du théorème. Pour conclure, les problèmes elliptiques aux conditions aux bords coercives possèdent la proprété de régularité elliptique a priori, et en particulier dans notre cas, une solution qui est $C^{2, \alpha}$ est automatiquement $C^{\omega}$.

Dans le cas du problème hydrodynamique, l'hypothèse (29) s'écrit

$$
\partial_{x_{n}}(g \cdot x)=g_{n} \neq 0,
$$

qui signifie que la surface $\Sigma$ ne soit pas de tangent verticale.

Revenant au problème (27) avec $G=\frac{1}{2}\left|\nabla_{x} \Phi\right|^{2}+g \cdot x-\frac{c^{2}}{2}$ provenant de la question originale des équations hydrodynamiques, le système linéarisé autour d'une solution de (26) est

$$
\left(\begin{array}{cc}
\partial_{y_{n}} & -\Xi_{y^{\prime}} \cdot \partial_{y^{\prime}} \\
\Xi_{y^{\prime}} \cdot \partial_{y^{\prime}} & g_{n}
\end{array}\right)\left(\begin{array}{c}
\xi \\
T
\end{array}\right)=0
$$

Il ne donne pas lieu à un problème coercif car son symbole principal est $\left(\Xi_{y^{\prime}} \cdot \xi^{\prime}\right)^{2}$ qui est dégénéré. Mais le symbole total est

$$
g_{n}\left|\xi_{n}\right|+\left(\Xi_{y^{\prime}} \cdot \xi^{\prime}\right)^{2}
$$

qui est positif dans le cas où $g_{n}>0$, à cause du signe du symbole sous-principal. Il est fort probable que la condition $\partial_{x_{n}} G(\cdot) 0$ implique la régularité a priori de la surface libre [7]. Cette condition sur le signe de $g$ s'interprète comme la condition physique que au point $q$, la surface $\Sigma$ est au dessus de l'eau dans la configuration en question.

\section{Références}

[1] W. Craig and A.-M. Matei. Regularity of the Neumann problem for free boundaries. in preparation (2003).

[2] D. Kinderlehrer and L. Nirenberg. Regularity in free boundary problems. Ann. Scuola Norm. Sup. Pisa Cl. Sci. Ser. IV 4, pp. 373-391 (1977).

[3] D. Kinderlehrer, L. Nirenberg and J. Spruck. Regularity in elliptic free boundary problems I. J. Analyse Math. 34, pp. 86-119 (1978).

[4] D. Kinderlehrer, L. Nirenberg and J. Spruck. Regularity in elliptic free boundary problems II; equations of higher order. Ann. Scuola Norm. Sup. Pisa Cl. Sci. Ser. IV 6, pp. 637-683 (1979).

[5] H. Lewy. A note on harmonic functions and a hydrodynamic application. Proc. AMS 3 pp. 111-113, (1952).

[6] A.-M. Matei. The Neumann problem for free boundaries in two dimensions.

C. R. Acad. Sci. Paris, Ser. I 335 pp. 1-6, (2002).

[7] G. Métivier. communication personelle, Forges-les-Eaux, 2-6 juin 2003. 
Department of Mathematics and Statistics

MCMASTER UNIVERSITY

Hamilton, Ontario L8P 4K1

CANADA

craig@math.mcmaster.ca

www.math.mcmaster.ca/craig/index.html

mateiam@math.mcmaster.ca

www.math.mcmaster.ca/matei 